\title{
Association of preoperative urethral parameters on magnetic resonance imaging and immediate recovery of continence following Retzius-sparing robot-assisted radical prostatectomy
}

\author{
Youjian $\mathrm{Li}^{1,2 \#}$, Weijian $\mathrm{Li}^{1,2 \#}$, Wenfeng $\mathrm{Lu}^{1,2}$, Mengxia Chen ${ }^{1,2}$, Jie Gao ${ }^{1,2}$, Yang Yang ${ }^{1,2}$, Junlong Zhuang ${ }^{1,2}$, \\ Xiaogong Li ${ }^{1,2}$, Hongqian Guo ${ }^{1,2}$, Xuefeng Qiu ${ }^{1,2}$ \\ ${ }^{1}$ Department of Urology, Drum Tower Hospital, Medical School of Nanjing University, Nanjing 210008, China; ${ }^{2}$ Institute of Urology, Nanjing \\ University, Nanjing 210008, China \\ Contributions: (I) Conception and design: H Guo, X Qiu, Y Li; (II) Administrative support: H Guo, X Qiu; (III) Provision of study material or \\ patients: W Li, X Li, Y Yang; (IV) Collections and assembly of data: M Chen, J Gao, W Lu; (V) Data analysis and interpretation: Y Li, J Zhuang; (VI) \\ Manuscript writing: All authors; (VII) Final approval of manuscript: All authors. \\ \#These authors contributed equally to this work. \\ Correspondence to: Xuefeng Qiu; Hongqian Guo. Department of Urology, Drum Tower Hospital, Medical School of Nanjing University, Nanjing \\ 210008, China. Email: Xuefeng_qiu@163.com; dr.ghq@nju.edu.cn.
}

Background: Studies regarding predictive factors of urinary continence following Retzius-sparing radical prostatectomy (RP) is limited. This study was designed to evaluate association of urethral parameters on preoperative magnetic resonance imaging (MRI) and immediate recovery of urinary continence following Retzius-sparing robot assisted radical prostatectomy (RS-RARP).

Methods: This retrospective cohort study enrolled 156 patients with clinically localized prostate cancer who underwent MRI before RS-RARP. We measured the following structures on preoperative MRI: minimal residual membranous urethral length (mRUL), peri-urethral sphincter complex (PSC) thickness, urethral wall thickness (UWT), the thicknesses of the levator ani muscle (LAM) and obturator internus muscle (OIM). Immediate urinary continence was defined as patients reported freedom from using safety pad within 7 days after removal of urinary catheter. Patients were divided into two groups according the median of each parameter on MRI. We retrospectively analyzed the patients in term of preoperative clinical factors and postoperative urinary continence.

Results: A total of 100 patients (64.1\%) reported immediate urinary continence after RS-RARP. Immediate urinary continence was significantly more in patients with longer mRUL $(\geq 8.70 \mathrm{~mm})$ than in patients with shorter mRUL ( $<8.70 \mathrm{~mm} ; \mathrm{P}=0.000)$. On multivariable analysis, longer mRUL was significantly related to immediate urinary continence after RS-RAPA (odds ratio 8.265; P=0.000). PSC, UWT, LAM and OIM were not associated with immediate urinary continence.

Conclusions: Our results firstly demonstrated that preoperative mRUL measured on MRI was an independent predictor of immediate urinary continence following RS-RARP. Therefore, preservation of membranous urethra is still the anatomical basis of better urinary outcome after RS-RARP.

Keywords: Immediate urinary continence; magnetic resonance imaging (MRI); prostate cancer; radical prostatectomy (RP); Retzius sparing

Submitted Sep 17, 2019. Accepted for publication Nov 29, 2019.

doi: $10.21037 /$ tau.2019.12.17

View this article at: http://dx.doi.org/10.21037/tau.2019.12.17 


\section{Introduction}

Urinary continence is one of main complications of radical prostatectomy (RP) (1). With a better understanding of the anatomy of prostate and its surrounding structures and improvement in technology and technical modifications, more than $80 \%$ of patients underwent RP could recovery urinary continence in 1 year after surgery (2). However, early recovery of urinary continence following RP is still poor, with more than $70 \%$ of patients requiring pads at 6 weeks after RP (2). Consequently, several surgical techniques have been described to improve early recovery of urinary continence after robot-assisted radical prostatectomy (RARP) (3). In 2010, Dr. Bocciardi and his colleagues firstly described Retzius-sparing robot-assisted radical prostatectomy (RS-RARP) (4). In this approach, anterior structures in Retzius space such as pubourethral ligaments, Santorini plexus could be preserved, therefore leading to better continence outcome early after RS-RARP (5). Recently, results from two randomized controlled trials further confirmed the effect of RS-RARP, providing level 1 evidence supporting an earlier return of urinary continence in patients underwent RS-RARP $(6,7)$.

Several preoperative factors have been shown to be associated with early recovery of continence after RARP. Biological patient-related factors, such as advanced age, higher BMI, lager prostate volume, and severe preoperative prostate symptom have been reported to be risk factor for continence outcome after RP (8). Furthermore, preoperative anatomic variables measured on magnetic resonance imaging (MRI), such as membranous urethra length (MUL) and periurethral supporting structures, have also been demonstrated to be associated with urinary continence outcome after RP (9). However, literature regarding predictive factors of urinary continence following RS-RARP is limited. The association between recovery of continence after RS-RARP and anatomic parameters on preoperative MRI is total unknown.

Therefore, this retrospective cohort study was designed to evaluate the association of perioperative urethral structures measured on MRI and recovery of immediate urinary continence after RS-RARP to identify possible predictive parameter for recovery of urinary continence. Given the recent wider application of multiparameter MRI (mpMRI) in diagnosis and staging of prostate cancer, the results of this study could provide urologists solid predictive information regarding urinary continence after RS-RARP, also better understanding the anatomic basis of RS-RARP in improving early recovery of urinary continence.

\section{Methods}

Between June 2017 and February 2019, patients with localized prostate cancer who underwent RS-RARP by the same surgeon (Dr. HG) at Nanjing Drum Tower Hospital were reviewed retrospectively. All Patients underwent multiparameter prostate MRI to evaluate extraprostatic extension and seminal vesicle invasion. Preoperative bone scan was applied to exclude the metastatic bone disease. Patients who with suspicious extraprostatic extension, seminal vesicle invasion, pelvic lymph nodes metastasis, or bone metastasis were excluded. Patients who had received neoadjuvant hormonal therapy or transurethral resection of the prostate were also excluded. Furthermore, patients with preoperative urinary incontinence were also excluded.

First, we calculated median values of each MRI parameter. According to median values of each MRI parameter, patients were divided into two groups. Differences in patient characteristics such as age, prostate volume, prostate-specific antigen (PSA), body mass index (BMI), preoperative international prostate symptom score (IPSS), nerve preservation, D’Amico risk group (10), biopsy Gleason score, positive surgical margin, extracapsular extension, seminal vesicle invasion and immediate urinary continence after RS-RARP between groups dichotomized by median values for each MRI parameter were analyzed. Next, univariable and multivariate analysis were performed to identify predictors associated with immediate urinary continence, including several factors likely to be related to postoperative immediate urinary continence, such as prostate volume, nerve preservation, preoperative IPSS, age, and BMI, and MRI parameters.

\section{Surgery and follow-up}

The technique of RS-RARP we used was similar to the transperitoneal approach described by Galfano et al. $(4,5)$ and Lim et al. (11). In patients with PSA $<10 \mathrm{ng} / \mathrm{mL}$, Gleason score $<7$, and clinical stages T1-2a, bilateral nerve was preserved. For nerve-sparing technique, bilateral intrafascial plane was undertaken according the surgical technique described by Galfano et al. (4). While those who did not meet the item did not retain the nerve. For patients at high risk of prostate cancer, extended lymph node dissection was performed. Adjuvant/salvage treatments, salvage radiotherapy is performed for patients with persistent PSA 
or biochemical recurrence. The catheter was removed between 7 and 10 days after operation, and discharged from hospital 3 to 5 days after operation. All patients were interviewed in the outpatient department every 3-month during the first year following RS-RARP to complete the questionnaire regarding urinary function or by telephone in case of missing questionnaires.

\section{MRI measurements and imaging}

The examinations were performed with a 3.0-T MR scanner (Ingenia, Philips Medical Systems, Best, The Netherlands). Patients were examined in the supine position. A 16-channel phased array surface body coil was used. Coronal T2-weighted images were obtained with the following parameters: repetition time (TR), 4,050 ms; echo time (TE), $90 \mathrm{~ms}$; slice thickness, $4 \mathrm{~mm}$; intersection gap, $0.4 \mathrm{~mm}$; field of view, $240 \times 240 \mathrm{~mm}$; matrix, $368 \times 285$. Sagittal T2-weighted imaging was performed with the following parameters: TR, 4,050 ms; TE, $90 \mathrm{~ms}$; slice thickness, $4 \mathrm{~mm}$; intersection gap, $0.4 \mathrm{~mm}$; field of view, $180 \times 180 \mathrm{~mm}$; matrix, 276×179. Transected T2-weighted images were obtained with the following parameters: TR, 4,750 ms; TE, $80 \mathrm{~ms}$; slice thickness, $3.5 \mathrm{~mm}$; intersection gap, $1 \mathrm{~mm}$; field of view, $220 \times 300 \mathrm{~mm}$; matrix, 276×226.

The sagittal T2-weighted FSE sequences allowed for minimal residual membranous urethral length (mRUL) to be measured parallel to the membranous urethra from the inferior edge of the levator ani muscle (LAM) to the superior margin of the bulbospongiosus muscle (12) (Figure 1A). Coronal T2-weighted sequences were used to measure peri-urethral sphincter complex (PSC) thickness, the thicknesses of the LAM, and obturator internus muscle (OIM) (Figure 1B). We measured PSC from the urethral midline to lateral margin of the converging LAM. We measured LAM from the extreme length converging on the urethra immediately caudal to the apex of prostate. And we measured OIM in its wider part. We measured membranous urethra in its wider part in the transected T2-weighted sequences before entering in the prostate and defined it as urethral wall thickness (UWT) (13) (Figure 1C). Measurements of the above MRI parameters were taken in a blind manner. All data were collected in centimeters with two decimal places.

\section{Continence evaluation}

We were used the Expanded Prostate Cancer Index
Composite (EPIC) instrument to evaluated urinary continence (14). All patients were considered as continence when they used no small safety liner or no pad at all. Immediate continence was defined as patients reported freedom from using any pad within 7 days after the urinary catheter was removed (6). All patients were subsequently reviewed within 7 days after the urinary catheter was removed to evaluate their urinary continence by accomplishing the self-administered questionnaire.

\section{Statistical analysis}

The Mann-Whitney U test was used for continuous data, and the chi-squared test or fisher exact test was used for categorical date. Continuous nonnormally distributed variables were presented as the median and interquartile range (IQR). Logistic regression analysis was applied for univariable and multivariable analysis to identify predictors of immediate urinary continence. All data analyses were performed using SPSS 21.0 statistical software (IBM SPSS, Chicago, IL, USA). A confidence interval (CI) of $95 \%$ was assumed, and a $\mathrm{P}$ value $<0.05$ was considered significant.

\section{Results}

Median values for mRUL, PSC, LAM, OIM, and UWT were $8.70,17.79,8.85,19.43$, and $10.42 \mathrm{~mm}$, respectively. Table 1 shows patients' basic characteristics compared between the two groups dichotomized by median values. $\mathrm{BMI} \geq 25 \mathrm{Kg} / \mathrm{m}^{2}$ rate was significantly lower in the groups with shorter PSC $(<17.79 \mathrm{~mm})(25.0 \%)$, LAM $(<8.85 \mathrm{~mm})$ (25.6\%) and UWT $(<10.42 \mathrm{~mm})(23.4 \%)$ than in the groups with longer PSC $(\geq 17.79 \mathrm{~mm})(47.5 \%)$, LAM $(\geq 8.85 \mathrm{~mm})$ (47.4\%) and UWT ( $\geq 10.42 \mathrm{~mm})(49.4 \% ; \mathrm{P}=0.004,0.005$, and 0.001 respectively). Patients with OIM $<19.43 \mathrm{~mm}$ were significantly older than those with OIM $\geq 19.43 \mathrm{~mm}$ $(\mathrm{P}=0.000)$, and the volume of prostate was larger than those with $\mathrm{OIM} \geq 19.43 \mathrm{~mm}(\mathrm{P}=0.030)$.

Follow-up data showed that there were 100 (64.1\%) patients reported freedom from using pad within 7 days after removal of urinary catheter. Table 1 also shows the rate of immediate urinary continence was significantly higher in the groups with longer mRUL than in the groups with shorter mRUL (rate: $80.8 \%$ vs. $47.4 \%, \mathrm{P}=0.000$ ). In contrast, no significant differences were found between longer and shorter PSC groups (rate: $63.7 \%$ vs. $64.5 \%$, $\mathrm{P}=0.925$ ), LAM groups (rate: $66.7 \%$ vs. $61.5 \%, \mathrm{P}=0.504$ ), OIM groups (rate: $66.7 \%$ vs. $61.5 \%, \mathrm{P}=0.504$ ), or UWT 

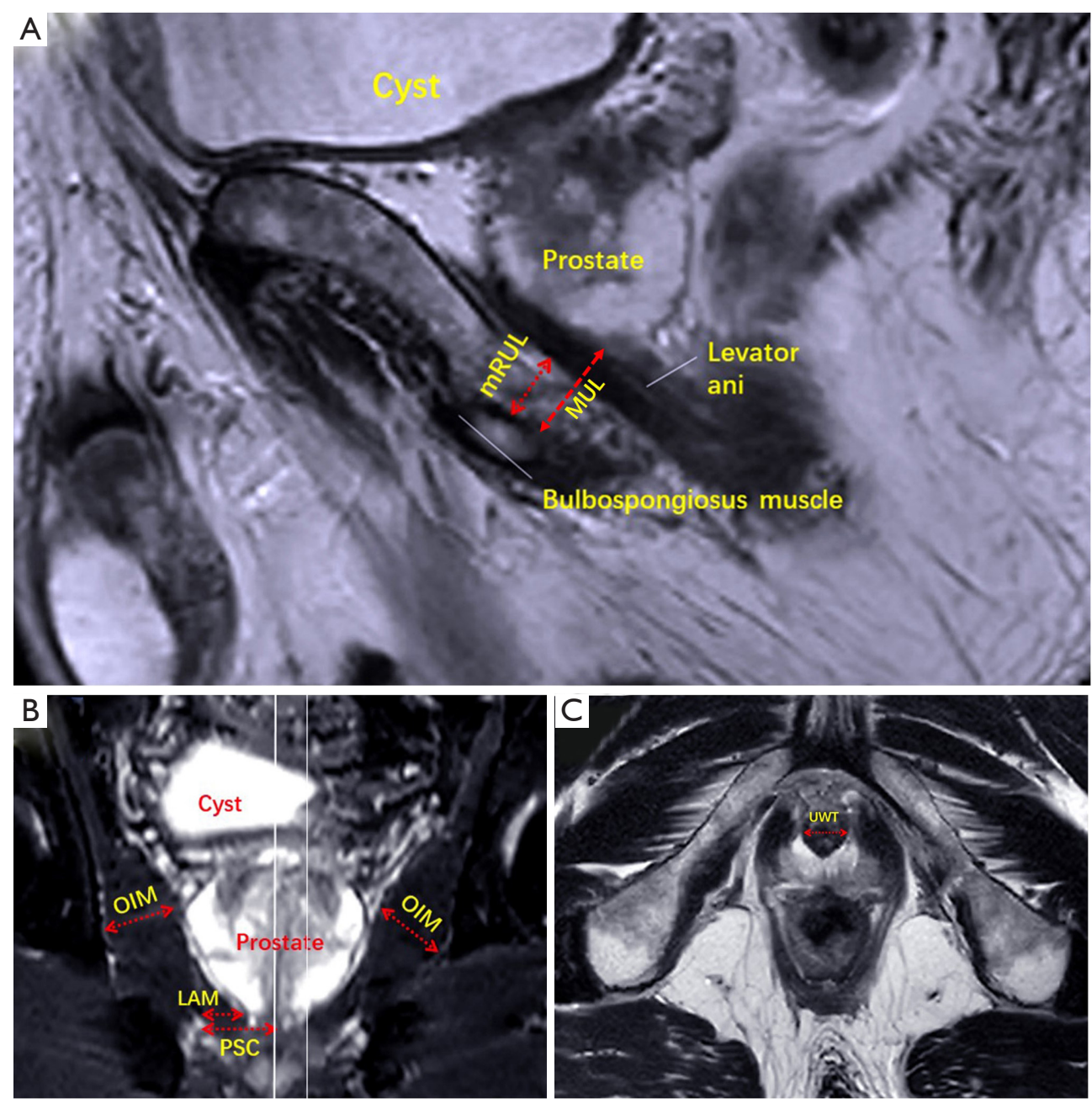

Figure 1 Measurements of magnetic resonance imaging (MRI). (A) Sagittal T2-weighted FSE sequences allowed for minimal residual membranous urethral length (mRUL) from the lower margin of the levator ani muscle (puboperinealis muscle) to the upper margin of the bulbospongiosus muscle in a direction parallel with the membranous urethra to be measured. Membranous urethra length (MUL) was measured parallel to the membranous urethra from the inferior edge of the prostate apex to the superior margin of the penile bulb; (B) coronal T2-weighted sequences were used to measure peri-urethral sphincter complex (PSC) thickness, the thicknesses of the levator ani muscle (LAM), and obturator internus muscle (OIM); (C) urethral wall thickness (UWT) was measured on transected T2-weighted sequences.

groups (rate: $65.8 \%$ vs. $62.3 \%, \mathrm{P}=0.650$ ).

On univariate regression analysis for the recovery of immediate urinary continence, longer mRUL [odds ratio $(\mathrm{OR})$ 4.654; $\mathrm{P}=0.000$ ] was significantly associated with the recovery of immediate urinary continence. Larger prostate volume (OR 0.979; $\mathrm{P}=0.032$ ), severe preoperative IPSS (OR 0.341; $\mathrm{P}=0.029)$, and older age (OR 0.895; $\mathrm{P}=0.000$; Table 2) were all significantly associated with delayed recovery (Table 2). On multivariate regression analysis, mRUL (OR 8.265; $\mathrm{P}=0.000$ ), severe IPSS (OR 0.245; $\mathrm{P}=0.018$ ), and age
(OR 0.897; $\mathrm{P}=0.001$; Table 2) were significant predictors of immediate urinary continence after RS-RARP (Table 2).

\section{Discussion}

The result of this retrospective cohort study revealed that the rate of postoperative immediate urinary continence in patients with longer mRUL was significantly higher than in those with shorter mRUL. Multivariate regression analysis also confirmed that mRUL was an independent predictor 


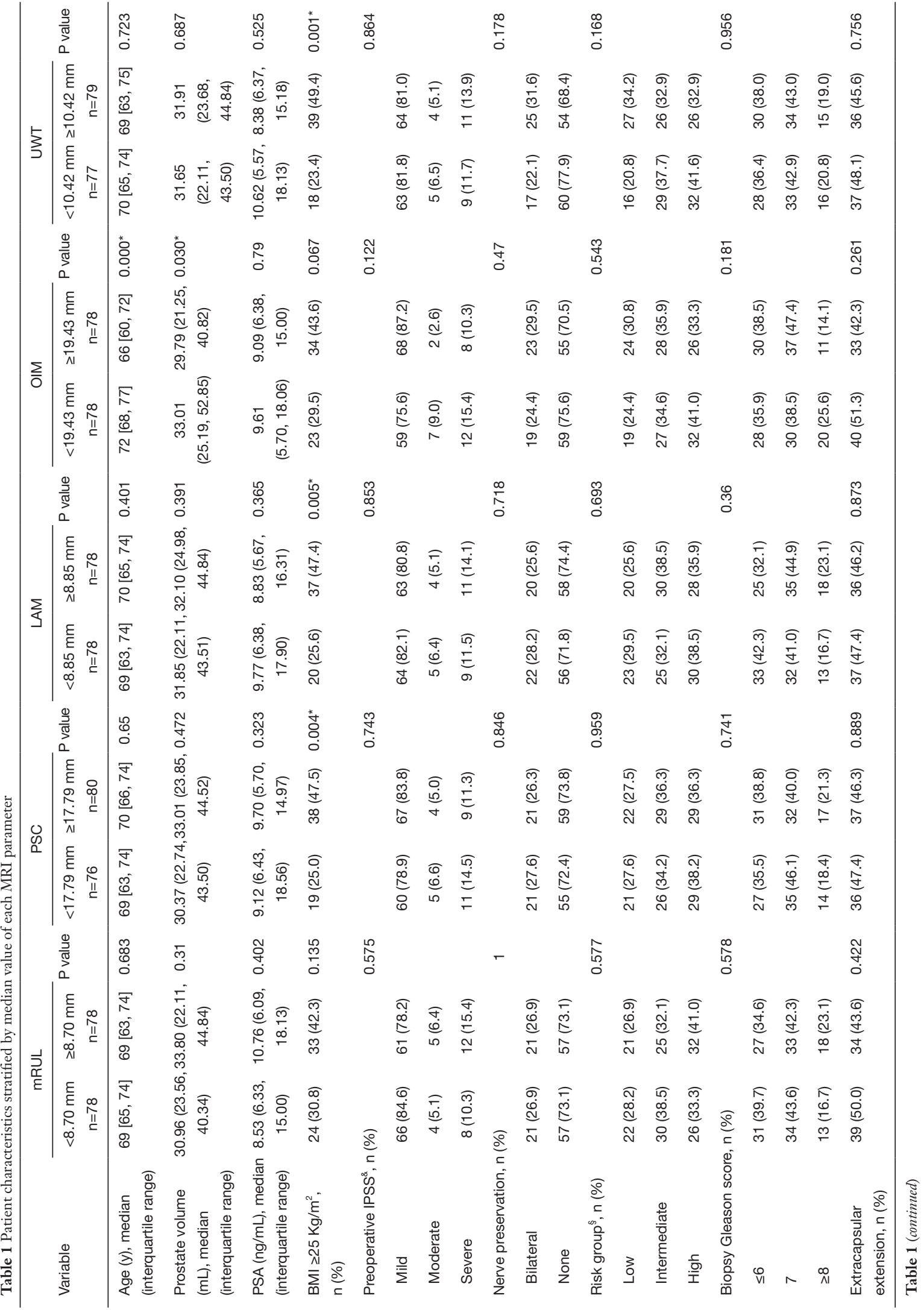




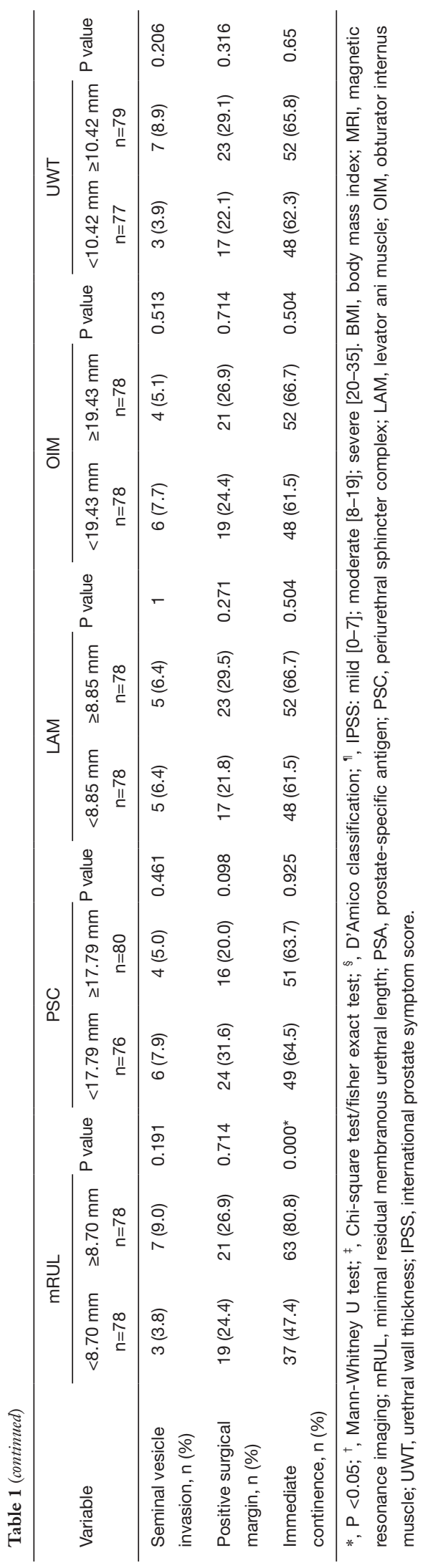

of postoperative immediate urinary continence. To the best of our knowledge, this is the first study to explore the association between urethral parameters on preoperative MRI and postoperative urinary outcome following RSRARP and to reveal that mRUL as an objective and quantitative predictor of immediate continence following RS-RARP.

RS-RARP was well demonstrated to be associated with improved recovery of early continence by several studies $(5-7,15)$. The updated results from one published randomized controlled trial showed that differences in urinary continence observed early after surgery were muted at 12 months follow-up (15). Therefore, in the present study, we set immediate urinary continence as the primary outcome as described in two randomized controlled trials $(6,7)$. In the present study, more than $50 \%$ of patients could achieve immediate continence after removal of catheter, revealing the advantage of RS-RARP in preserving urinary function. However, there is still part of patients could not return to immediate continence after surgery, leading to the anxiousness of patients due to the lack of information on predicting the status and duration of urinary incontinence. Therefore, it is important to identify possible predictors of continence early after RS-RARP, which could relieve the anxiousness of patients, as well as offer prognostic information to surgeons when counselling patients in clinical practice prior to surgery and when explaining a delay in continence recovery following surgery.

Given the recent advances that have led to the wider application of MRI for diagnosis and clinical staging of prostate cancer (16), many studies have shown the great correlation between anatomic variables such as preoperative MUL and periurethral supporting structures. In particularly, longer MUL has been considered to be significantly associated with better postoperative continence recovery following RP $(13,17,18)$. MUL has been considered to be a prognostic risk factor for overall continence recovery including the early recovery after RP (9). Compare to those biological factors for predicting urinary continence, MUL is more objective and easy to quantify. Therefore, a comprehensive understanding of MUL is potentially of value to predict urinary continence after RP. However, there is no standardized method to measure MUL because it's both boundaries are unclear on MRI. Furthermore, part of MUL would be damage during surgery. Therefore, Satake et al. proposed mRUL as a new parameter to reflect MUL (12). Compared to MUL, mRUL has clear boundaries on MRI, from the lower margins of the 
Table 2 Logistics regression analysis of immediate urinary continence

\begin{tabular}{|c|c|c|c|c|c|c|c|c|}
\hline \multirow{2}{*}{$\begin{array}{l}\text { Variable } \\
\text { mRUL (mm) }\end{array}$} & \multicolumn{4}{|c|}{ Univariate analysis } & \multicolumn{4}{|c|}{ Multivariate analysis } \\
\hline & $\frac{\text { OR }}{4.654}$ & 2.271 & 9.536 & $\frac{P}{0.000^{*}}$ & $\begin{array}{c}\text { OR } \\
8.265\end{array}$ & 3.359 & 20.339 & $\frac{P}{0.000^{*}}$ \\
\hline Prostate volume (mL) & 0.979 & 0.961 & 0.998 & $0.032^{*}$ & 0.981 & 0.959 & 1.004 & 0.107 \\
\hline Nerve preservation & 1.571 & 0.729 & 3.388 & 0.249 & 1.186 & 0.466 & 3.023 & 0.720 \\
\hline Preoperative IPSS & & & & $0.032^{*}$ & & & & $0.022^{*}$ \\
\hline Mild & 1 & \multicolumn{2}{|c|}{ Referent } & - & 1 & \multicolumn{2}{|c|}{ Referent } & - \\
\hline Moderate & 4.095 & 0.496 & 33.815 & 0.191 & 4.055 & 0.431 & 38.191 & 0.221 \\
\hline Severe & 0.341 & 0.130 & 0.898 & $0.029^{*}$ & 0.245 & 0.077 & 0.784 & $0.018^{*}$ \\
\hline
\end{tabular}

*, $\mathrm{P}<0.05$. $\mathrm{Cl}$, confidence interval; OR, odds ratio; mRUL, minimal residual membranous urethral length; BMI, body mass index; IPSS, international prostate symptom score.

levator ani to upper margin of the bulbospongiosus muscles (Figure 1). Also, mRUL usually could be preserved without damage during surgery. Therefore, we applied mRUL in this study as a possible predictor of urinary continence following RS-RARP. The results showed that mRUL can be used as a novel predictor of immediate urinary continence after RS-RARP. It is easy to speculate that patients with longer mRUL can retain a complete longer urethral sphincter, resulting in high urethral closure pressure after RS-RARP (9). In the first paper describing RS-RARP, Dr. Bocciardi and his colleagues proposed that better urinary continence outcome following RS-RARP was theoretically due to the preservation of anatomical structures, such as Aphrodite's veil, Santorini plexus, and pubourethral ligaments (4). From the results of the present study, it revealed that preservation of membranous urethra is still the basis of better urinary continence outcome. There was no significant difference in the rate of postoperative immediate urinary continence between long and short groups of other MRI parameters, indicated that they may have no correlation with immediate urinary continence after RSRARP.

Furthermore, we found that severe preoperative IPSS and advanced age were important factors affecting immediate urinary continence recovery after RS-RARP in the multivariate regression analysis. Preoperative prostate symptom and age have been well demonstrated to be biological factors affecting urinary continence recovery after RP (19). Some studies have shown that patients with lower preoperative IPSS recover more quickly after surgery $(20,21)$. It has been hypothesized that severe preoperative IPSS may be due to overactivity of detrusor caused by benign prostatic hyperplasia, which may delay the recovery of continence after RP (22). It is reported that increased age is associated with the inferior continence recovery after RP $(23,24)$. The density of striated muscle cells decreases with age (25), which may be the main reason for the increased incidence of urinary incontinence with advancing age after surgery.

In the present study, we found that PSC, LAM and UWT are all associated with BMI, but no reports of these could be found in the literature. So we don't know the underlying mechanism. Also, we found patients with OIM $<19.43 \mathrm{~mm}$ were older than those with OIM $\geq 19.43 \mathrm{~mm}$, and the volume of prostate was larger than those with OIM $\geq 19.43 \mathrm{~mm}$. It has been hypothesized that the OIM atrophies with age (26), and the volume of the prostate increases with age (27). That might be able to explain patients with OIM $<19.43 \mathrm{~mm}$ had greater age that those with $\mathrm{OIM} \geq 19.43 \mathrm{~mm}$.

According to the current literature, positive surgical margin is $15.6 \%$ for pT2 cases, while $38.53 \%$ for patients with pT3 disease (28). In the present study, $46 \%$ patients were with extracapsular extension confirmed by postoperative histopathology (Table 1), which could explain the higher positive surgical margin $(\sim 25 \%)$ in our cohort. 
Several limitations need to be considered in this study. First, this was a single-institution research, and the cohort was comparatively small. Second, we used patient-reported pad usage to define the state of urinary continence, which was not an objective quantitative measurement. Finally, although surgery was performed by the same experienced surgeon using the same technique, the nuance of surgical techniques in each operation might have affected postoperative urinary continence. However, we believe that our study correctly reflect the importance of mRUL for immediate urinary continence after RS-RARP. When measuring the parameters by MRI, we did not know the patient's postoperative urinary continence state, so there was no bias. Nevertheless, multicenter prospective studies are needed to validate the repeatability of our results.

\section{Conclusions}

In conclusion, the present study revealed that preoperative mRUL measured on MRI was an independent predictor of immediate urinary continence following RS-RARP. Combined with age and preoperative prostate symptom score, mRUL could give physicians important predictive information of immediate recovery of urinary continence after RS-RARP.

\section{Acknowledgments}

Funding: This study was supported by grants from the National Natural Science Foundation of China (81602232, 81772710, 81602221), the Project of Invigorating Health Care through Science, Technology and Education, Jiangsu Provincial Key Medical Discipline (Laboratory) (ZDXKB2016014), National Natural Science Foundation of Jiangsu Province (BK20160117), Nanjing Medical Science and technique Development Foundation (QRX17128) and Nanjing Health Distinguished Youth Fund (JQX16025).

\section{Footnote}

Conflicts of Interest: All authors have completed the ICMJE uniform disclosure form (available at http://dx.doi. org/10.21037/tau.2019.12.17). The authors have no conflicts of interest to declare.

Ethical Statement: The authors are accountable for all aspects of the work in ensuring that questions related to the accuracy or integrity of any part of the work are appropriately investigated and resolved. All procedures performed in this study were in accordance with the Declaration of Helsinki and approved by the Ethics Committee of the Drum Tower Hospital, Medical School of Nanjing University. Because of the retrospective nature of the research, the requirement for informed consent was waived.

Open Access Statement: This is an Open Access article distributed in accordance with the Creative Commons Attribution-NonCommercial-NoDerivs 4.0 International License (CC BY-NC-ND 4.0), which permits the noncommercial replication and distribution of the article with the strict proviso that no changes or edits are made and the original work is properly cited (including links to both the formal publication through the relevant DOI and the license). See: https://creativecommons.org/licenses/by-nc$\mathrm{nd} / 4.0 \%$.

\section{References}

1. Salonia A. Words of wisdom. Re: Quality of life and satisfaction with outcome among prostate-cancer survivors. Eur Urol 2009;55:526-7.

2. Ficarra V, Novara G, Rosen RC, et al. Systematic review and meta-analysis of studies reporting urinary continence recovery after robot-assisted radical prostatectomy. Eur Urol 2012;62:405-17.

3. Sridhar AN, Abozaid M, Rajan P, et al. Surgical Techniques to Optimize Early Urinary Continence Recovery Post Robot Assisted Radical Prostatectomy for Prostate Cancer. Curr Urol Rep 2017;18:71.

4. Galfano A, Ascione A, Grimaldi S, et al. A new anatomic approach for robot-assisted laparoscopic prostatectomy: a feasibility study for completely intrafascial surgery. Eur Urol 2010;58:457-61.

5. Galfano A, Di TD, Sozzi F, et al. Beyond the learning curve of the Retzius-sparing approach for robot-assisted laparoscopic radical prostatectomy: oncologic and functional results of the first 200 patients with $\geq 1$ year of follow-up. Eur Urol 2013;64:974-80.

6. Dalela D, Jeong W, Prasad MA, et al. A Pragmatic Randomized Controlled Trial Examining the Impact of the Retzius-sparing Approach on Early Urinary Continence Recovery After Robot-assisted Radical Prostatectomy. Eur Urol 2017;72:677-85.

7. Asimakopoulos AD, Topazio L, De Angelis M, et al. Retzius-sparing versus standard robot-assisted radical 
prostatectomy: a prospective randomized comparison on immediate continence rates. Surg Endosc 2019;33:2187-96.

8. Qin H, Qiu X, Ma H, et al. Predictors for immediate recovery of continence following Retzius-sparing robotassisted radical prostatectomy: a case-control study. Int Urol Nephrol 2019;51:825-30.

9. Mungovan SF, Sandhu JS, Akin O, et al. Preoperative Membranous Urethral Length Measurement and Continence Recovery Following Radical Prostatectomy: A Systematic Review and Meta-analysis. Eur Urol 2017;71:368-78.

10. D'Amico AV, Whittington R, Malkowicz SB, et al. Biochemical outcome after radical prostatectomy or external beam radiation therapy for patients with clinically localized prostate carcinoma in the prostate specific antigen era. Cancer 2002;95:281-6.

11. Lim SK, Kim KH, Shin TY, et al. Retzius-sparing robotassisted laparoscopic radical prostatectomy: combining the best of retropubic and perineal approaches. Bju International 2014;114:236-44.

12. Satake Y, Kaiho Y, Saito H, et al. Estimated Minimal Residual Membranous Urethral Length on Preoperative Magnetic Resonance Imaging Can be a New Predictor for Continence after Radical Prostatectomy. Urology 2018;112:138-44.

13. Tienza A, Hevia M, Benito A, Pascual JI, Zudaire JJ, Robles JE. MRI factors to predict urinary incontinence after retropubic/laparoscopic radical prostatectomy. Int Urol Nephrol 2015;47:1343-9.

14. Wei JT, Dunn RL, Litwin MS, et al. Development and validation of the expanded prostate cancer index composite (EPIC) for comprehensive assessment of health-related quality of life in men with prostate cancer. Urology 2000;56:899-905.

15. Menon M, Dalela D, Jamil M, et al. Functional Recovery, Oncologic Outcomes and Postoperative Complications after Robot-Assisted Radical Prostatectomy: An EvidenceBased Analysis Comparing the Retzius Sparing and Standard Approaches. J Urol 2018;199:1210-7.

16. Duvnjak P, Schulman AA, Holtz JN, et al. Multiparametric Prostate MR Imaging: Impact on Clinical Staging and Decision Making. Urol Clin North Am 2018;45:455-66.

17. Paparel P, Akin O, Sandhu JS, et al. Recovery of Urinary Continence after Radical Prostatectomy: Association with Urethral Length and Urethral Fibrosis Measured by Preoperative and Postoperative Endorectal Magnetic
Resonance Imaging. Eur Urol 2009;5 5:629-37.

18. Hakimi AA, Faleck DM, Agalliu I, et al. Preoperative and intraoperative measurements of urethral length as predictors of continence after robot-assisted radical prostatectomy. J Endourol 2011;25:1025-30.

19. Heesakkers J, Farag F, Bauer RM, et al. Pathophysiology and Contributing Factors in Postprostatectomy Incontinence: A Review. Eur Urol 2017;71:936-44.

20. Lavigueur-Blouin H, Noriega AC, Valdivieso R, et al. Predictors of early continence following robot-assisted radical prostatectomy. Can Urol Assoc J 2015;9:e93-7.

21. Tienza A, Hevia M, Merino I, et al. Can low urinary tract symptoms influence postprostatectomy urinary incontinence? Minerva Urol Nefrol 2016;68:324-9.

22. Shikanov S, Desai V, Razmaria A, et al. Robotic radical prostatectomy for elderly patients: probability of achieving continence and potency 1 year after surgery. J Urol 2010;183:1803-7.

23. Namiki S, Ishidoya S, Tochigi T, et al. Quality of life after radical prostatectomy in elderly men. Int J Urol 2009;16:813-9.

24. Anderson CB, Kaufman MR, Dietrich MS, et al. Recovery of Urinary Function After Radical Prostatectomy: Identification of Trajectory Cluster Groups. J Urol 2012;187:1346-51.

25. Strasser H, Tiefenthaler M, Steinlechner M, et al. Urinary incontinence in the elderly and age-dependent apoptosis of rhabdosphincter cells. Lancet 1999;354:918-9.

26. Cook MS, Bou-Malham L, Esparza MC, et al. Agerelated alterations in female obturator internus muscle. Int Urogynecol J 2017;28:729-34.

27. Xia SJ, Xu XX, Teng JB, et al. Characteristic pattern of human prostatic growth with age. Asian J Androl 2002;4:269-71.

28. Checcucci E, Veccia A, Fiori C, et al. Retzius-sparing robot-assisted radical prostatectomy vs the standard approach: a systematic review and analysis of comparative outcomes. BJU Int 2020;125:8-16.

Cite this article as: $\mathrm{Li} \mathrm{Y,} \mathrm{Li} \mathrm{W,} \mathrm{Lu} \mathrm{W,} \mathrm{Chen} \mathrm{M,} \mathrm{Gao} \mathrm{J,} \mathrm{Yang} \mathrm{Y,}$ Zhuang J, Li X, Guo H, Qiu X. Association of preoperative urethral parameters on magnetic resonance imaging and immediate recovery of continence following Retzius-sparing robot-assisted radical prostatectomy. Transl Androl Urol 2020;9(2):501-509. doi: 10.21037/tau.2019.12.17 